\title{
Infarction and shrinkage of a meningioma after extensive cosmetic surgery
}

Spontaneous infarction in meningiomas causing acute symptoms is rare. Treating physicians should be aware of the phenomenon of spontaneous infarction in brain tumours after prolonged surgery, presumably related to hypotension or anaemia.

A 43 year old obese woman had MRI in 1998 for nondescript headache; it showed an incidental torcular meningioma (figure A). Because the lesion did not explain her symptoms, conservative management was elected. The patient underwent gastroplasty, proceeded to lose $36 \mathrm{~kg}$, and then underwent circumferential abdominoplasty and rhytidectomy in early 1999. As part of her body-contouring surgery, she underwent mammary reduction, axillary excision, brachioplasty, and leg dermal lipectomies, in late 1999. This operation lasted for 8 hours and a total of 1650 $\mathrm{g}$ tissue was removed; brief episodes of hypotension were documented. Two days postoperatively she developed severe headache, nausea, and vomiting. Brain MRI showed a central area of presumed necrosis but no change in overall tumour size or peritumoral oedema (figure B). With conservative treatment she returned to her baseline status and 10 months later she remained well and MRI showed significant reduction in tumour size (figure C). The patient remained on oral iron supplements for anaemia.

Penfield was the first to describe ischaemic changes in meningiomas. ${ }^{1} \mathrm{He}$ thought that meningiomas have a peripheral blood supply and that the centre of the tumour is a "watershed area" which becomes increasingly oligaemic as the tumour grows. Pullicino et al reported a case of central infarction occurring within a frontal meningioma after a short period of hypotension during cardiac arrest. ${ }^{2}$

Necrosis in a meningioma after systemic chemotherapy has also been reported ${ }^{3}$; the patient had a rectal carcinoma
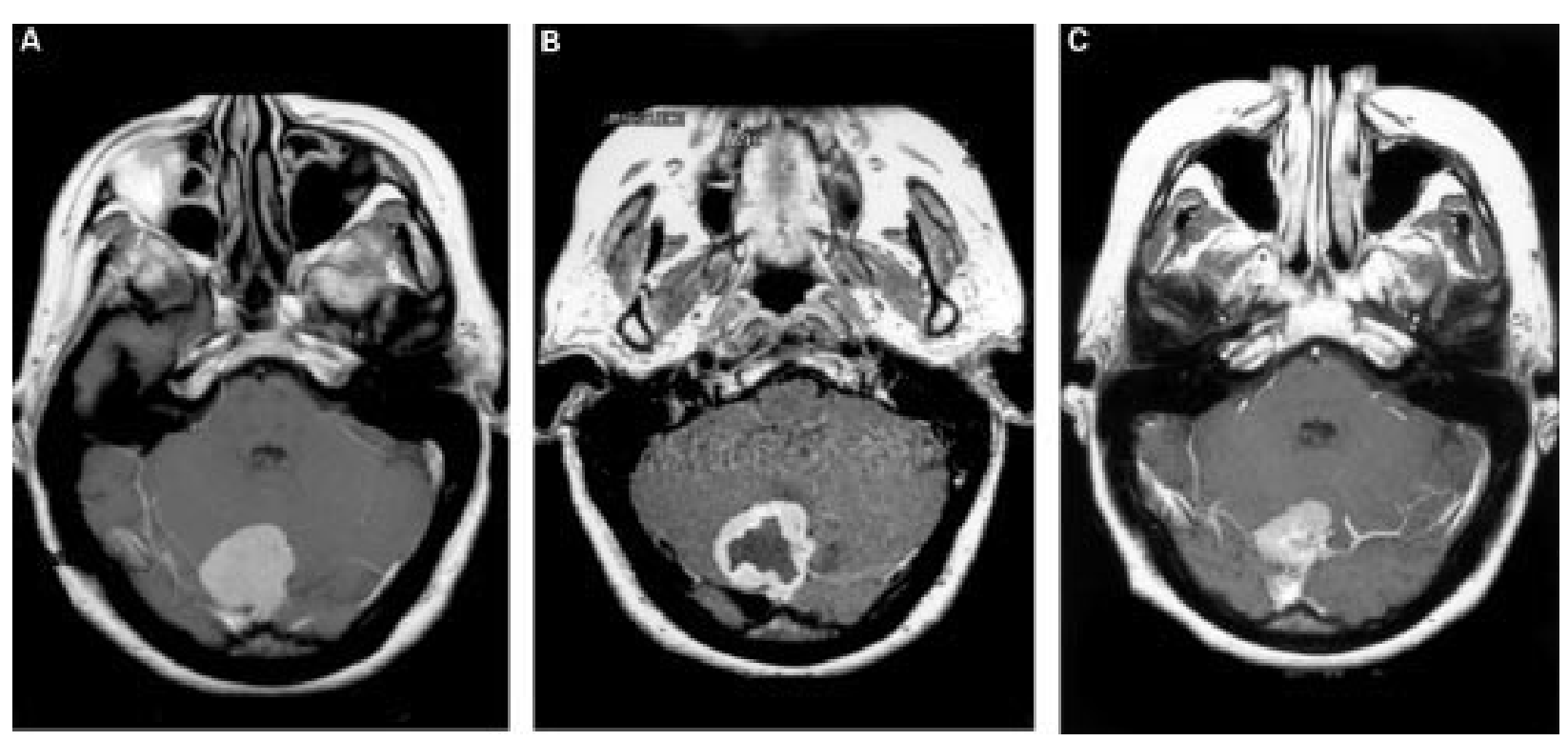

(A) Enhanced T1 weighted axial MRI of the patient when she was initially seen by a neurosurgeon. (B) Enhanced T1 weighted axial MRI of the patient 3 days after extensive body-contouring surgery. (C) Enhanced T1 weighted axial MRI of the patient 10 months later. and an asymptomatic large vertex meningioma. He presented 2 weeks after the start of chemotherapy for his rectal tumour with neurological deterioration necessitating surgical removal of his brain tumour. A large area of central necrosis was seen on imaging and pathological examination of the tumour disclosed a typical syncytial meningioma with large areas of necrosis of at least 1 week of age.

Our patient presented with severe headache on the 3rd day after extensive cosmetic surgery that presumably caused infarction in the centre of the tumour due to hypotension from hypovolaemia; the centre of the tumour would be expected to be more vulnerable to infarction from hypoperfusion. The infarction probably caused minimal enlargement of the tumour, which led to increased venous pressure and increased intracranial pressure. Almost a year later MRI showed involution of the necrotic portion of the tumour and overall decrease in tumour size. In this case the clinical symptoms and imaging changes in the tumour were temporally related to extensive elective cosmetic surgery but there is still the possibility that the tumour infarction occurred spontaneously.

K B BALKHOYOR $M$ BERNSTEIN

Division of Neurosurgery, Toronto Western Hospital, 399 Bathurst Street, Suite 2-405 McL, University of Toronto, Toronto, Ontario M5T 2S8,

Canada

Correspondence to: Dr M Bernstein mark.bernstein@uhn.on.ca

1 Penfield W. Tumors of the sheaths of the nervous system. In Penfield W, ed. Cytology and cellular pathology of the nervous system. Vol 3. New York: PB Hoeber, 1932:955-89.

2 Pullicino P, Wilbur DC, Levy RJ, et al. Infarction in a meningioma after cardiac arrest. Arch Neurol 1983;40:456-7.

3 Bernstein M, Villamil A, Davidson G, et al. Necrosis in a meningioma Bernstein M, Villamil A, Davidson G, et al. Necrosis in a meningioma
following systemic chemotherapy. Case report. $\mathcal{F}$ Neurosurg $1994 ; 81: 284-7$. 